\title{
Spondylodiscitis and endocarditis caused by S. vestibularis
}

\begin{abstract}
Streptococcus vestibularis is a recently described member of the viridans group that was first isolated from the vestibular mucosa of the human oral cavity and described as a new species in 1988. It has been rarely associated with human infections. In few papers, it has been reported as a causal agent of systemic infection in immunosupressed adults and in those with other severe underlying diseases, like coronary valve diseases. A 65-year-old woman was admitted to the hospital with complaints of fever for three months, general malaise, effort dyspnea, weight loss, back pain and myalgia. Both native aortic valve endocarditis and spondylodiscitis due to Streptococcus vestibularis were detected. The patient was successfully treated with intravenous potassium penicillin $G$ and gentamicin for six weeks, followed by oral amoxicillin for three months, in addition to aortic valve replacement. In all patients with spondylodiscitis, infective endocarditis should be considered, particularly in patients with heart valve disease history, since spondylodiscitis may be the presenting sign of an infective endocarditis. Cardiac valve replacement surgery should be performed if the course of fever and inflammatory syndrome is unfavorable after appropriate antibiotic treatment. We report the first case with both native aortic valve endocarditis and spondylodiscitis due to Streptococcus vestibularis.
\end{abstract}

Keywords: endocarditis, spondylodiscitis, Streptococcus vestibularis.

[Braz J Infect Dis 2010;14(4):377-379]@Elsevier Editora Ltda.

\section{INTRODUCTION}

Streptococcus vestibularis is a member of the salivarious group of viridans streptococci. Many of the viridans streptococcal species are part of human normal microbial flora. The commensal species are most prevalent in the oral cavity. S. vestibularis has been accepted as an important nosocomial pathogen and an atypical cause of significant septicemia in the presence of mucosal lesions, immune compromising states, malignancy, liver cirrhosis, renal failure or chronic diseases, such as diabetes mellitus. $S$. vestibularis has been reported to be associated with severe human infections, such as meningitis, endocarditis, bacteremia, and bone and joint infections. ${ }^{1-4}$ Infective endocarditis (IE) occurs in older age group (over the age of 60) who has prosthetic valves or structural heart diseases. Few of them have underlying rheumatic valve disease or intravenous drug abuse. Staphylococcus aureus and viridans streptococci are the most common causes of native valve infective endocarditis. Usually, more than 50\% of all cases of IE are caused by viridans streptococci. Many members of the viridans family are known to be able to cause IE, including Streptococcus mitis, Streptococcus sanguis, Streptococcus mutans, Streptococcus salivarus and Streptococcus oralis. New etiological agents have been recently described. ${ }^{5,6}$ Other members of the viridans group are also able to cause IE, but poorly defined taxonomy and difficulties in specification prevent this ascertainment from being made. Few cases of prosthetic valve IE due to S. vestibularis were reported. ${ }^{7}$ The only case of native valve endocarditis due to S. vestibularis was reported as a coinfection with S. oralis. ${ }^{6}$ In the literature, there is no case report about native valve endocarditis caused by S. vestibularis as a single causative pathogen. Less than $4 \%$ of patients with endocarditis actually demonstrate spondylodiscitis. ${ }^{8}$ Spondylodiscitis is a prolonged inflammation of two adjacent vertebral bodies and the disc between them. Due to the avascular nature of the intervertebral disc space in adults, spondylodiscitis is rarely observed. Eighty percent of spondylo-
Authors

Muge Aydin Tufan, MD ${ }^{1}$

Kart-Koseoglu Hamide, Associate Professor ${ }^{2}$

Ersozlu-Bozkirli Duygu, $\mathrm{MD}^{1}$

Azap Ozlem, Associate Professor $^{2}$

Tufan Kadir, MD ${ }^{3}$

Yucel Ahmet Eftal,

Professor $^{1}$

${ }^{1}$ Division of Rheumatology, Baskent University Faculty of Medicine, Ankara,

Turkey.

${ }^{2}$ Department of Clinical Microbiology and

Infectious Disease, Baskent University Faculty of

Medicine, Ankara, Turkey. ${ }^{3}$ Department of

Neurosurgery, Baskent

University Faculty of

Medicine, Ankara, Turkey.

Submitted on: 03/04/2010 Approved on: 04/06/2010

Correspondence to:

Muge Aydin Tufan, MD Baskent Universitesi Adana Uygulama ve Araştırma Merkezi Dadaloglu Mah. 39 Sk. No:6 01250 - Adana - Turkey Phone: +90-533-2333476 Fax: +90-322-3271274

E-mail: mugeaydin@ yahoo.com

We declare no conflict of interest. 
discitis were hematogenous, and 20\% developed after spinal surgery. ${ }^{8}$ Potential sources of hematogenous or contiguous spread of infection include the genitourinary tract, skin and soft tissue, respiratory tract, infected intravenous catheters, postoperative wound infections, endocarditis, and dental infections. However, the primary site of infections cannot be identified in most of cases. Less common causes include infected native or prosthetic valves. ${ }^{9}$ Staphylococcus aureus is the most frequent cause of spondylodiscitis, followed by Streptococcus spp, Escherichia coli and Mycobacterium tuberculosis..$^{10}$ Until this report, there has been no previous report of spondylodiscitis and native valve endocarditis due to $S$. vestibularis in the literature.

\section{CASE REPORT}

A 65-year-old woman was admitted to the hospital with complaints of fever for three months, general malaise, effort dyspnea, weight loss, back pain and myalgia. From her medical history it was learned that she had hypertension for three years. She was on atenolol $50 \mathrm{mg}$ daily. She had suddenly started to feel back pain three months previously, without trauma or brisk movement. The pain worsened with movement and she was admitted to another hospital with these complaints. Her laboratory tests were unremarkable, except for elevated C-reactive protein (CRP) $(77 \mathrm{mg} / \mathrm{L})$ and erythrocyte sedimentation rate (ESR) $(37 \mathrm{~mm} / \mathrm{h})$. Chest radiography revealed elevated density on the bilateral inferior zones. Chest computed tomography showed mild septal lines and pleural thickening. Atypical pneumonia was considered and the patient was put on $400 \mathrm{mg}$ of moxifloxacin intravenously, daily, for two weeks. After cessation of antibiotic therapy, there was a mild decrease of acute phase proteins and she was subsequently discharged. One month later, she was admitted to our hospital with low back pain, fever, high CRP $(220 \mathrm{mg} / \mathrm{L})$ and ESR $(90 \mathrm{~mm} / \mathrm{h})$ levels. Palpation and percussion of the $6-7^{\text {th }}$ thoracic vertebrae revealed tenderness. The neurological examination was normal. Complete blood count and biochemical analyses were within normal limits. During the first few days of hospitalization, she had fever $\left(39^{\circ} \mathrm{C}\right)$ and a $2 / 6$ pansystholic murmur on physical examination. Blood cultures were taken. The plain radiography of thoracic vertebra was normal. Magnetic resonance imaging (MRI) showed pathological enhancement on intervertebral disc space and neighboring endplates, considering spondylodiscitis at 6-7 th thoracic intervertebral disc space (Figure 1). Bone scintigraphy demonstrated high activity in T7 vertebra body. Serum brucella agglutination test was negative and tuberculin skin test was found to be negative (induration $<5 \mathrm{~mm}$ ). The electrocardiogram revealed sinus tachycardia. Transthoracic and transesophageal echocardiography revealed two calcific mobile vegetations $(1.1 \times 0.7 \mathrm{~cm}, 1.6 \mathrm{x}$ $0.8 \mathrm{~cm}$ ) on the aortic valve, compatible with endocarditis. A computed tomography guided needle biopsy of the interver-
Figure 1: Sagittal precontrast (a) and postcontrast (b) T1 weighted MR images show enhancement of the T6-7 intervertebral disc space and adjacent vertebrae endplates.

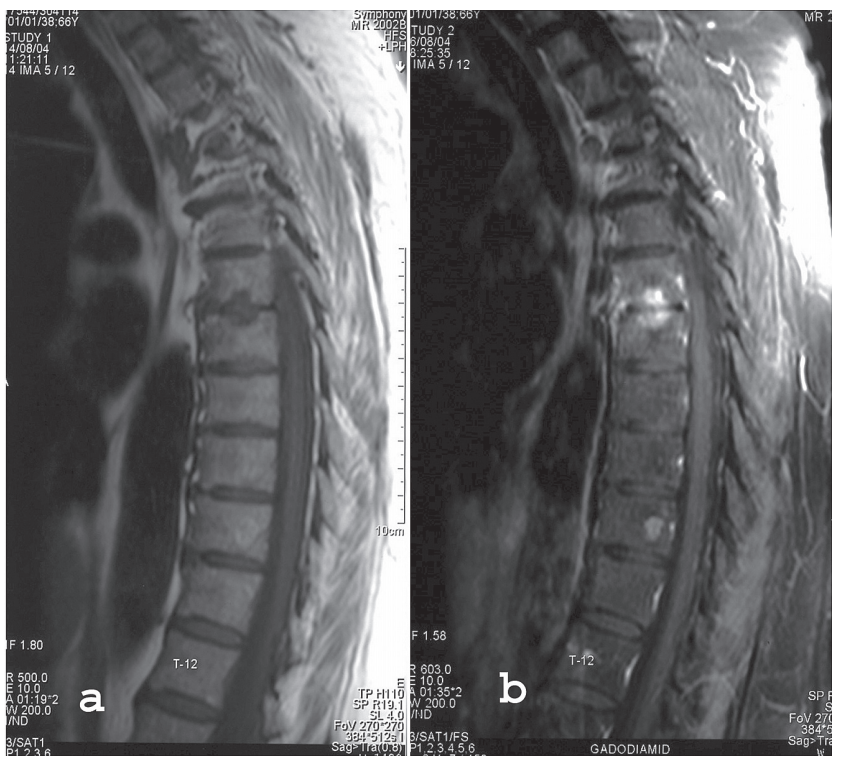

tebral space at T6-7 was performed. S. vestibularis was grown both in blood cultures taken on the first hospitalization day and in the culture of fine needle aspiration specimen of intervertebral space. Bone marrow cultures were also positive for the same agent. The patient received $6 \times 4$ million units of potassium penicillin G daily intravenously and $240 \mathrm{mg} /$ daily gentamicin intravenously for six weeks. Although fever resolved, high CRP level, effort dyspnea and tachycardia persisted. Since the endocardial lesions showed progression, she underwent aortic valve replacement. The patient recovered after operation and was discharged from hospital. Three grams of oral amoxicillin daily was administrated for three months. Control MRI showed no evidence of inflammation on T6-7 intervertebral space. Blood cultures were negative. Three years follow up was uneventful.

\section{DISCUSSION}

Oral streptococci are among the most common causes of infective endocarditis. S. vestibularis is a newly described species within the viridans streptococci group. ${ }^{11}$ S. vestibularis is an extremely rare cause of bacteremia and endocarditis, especially in older persons or in patients who have underlying chronic disease. In our case, except for being elderly, there were no risk factors, such as diabetes mellitus, malignancy, use of immunosuppressant, operation and/or rheumatoid arthritis.

Spondylodiscitis is rarely observed in association with infective endocarditis. Le Moal et al. ${ }^{12}$ reported that the prevalence of spondylodiscitis in patients with IE was of $15 \%$. Spondylodiscitis, however, has rarely been described, partic- 
ularly in case reports. Ninet et al. ${ }^{13}$ found the prevalence of spondylodiscitis in patients with IE to be of $5.9 \%$, whereas Thomas et al. ${ }^{14}$ reported a prevalence of $3.7 \%$. Spondylodiscitis does not appear to worsen the prognosis of IE, although the need for cardiac valve replacement seems to be more frequent in IE patients with spondylodiscitis. Presenting symptoms are variable and sometimes may delay the diagnosis. The mean duration of symptoms from onset to diagnosis ranges from two days to six months. A careful review of the patient's history and findings of physical examination, as well as laboratory data and diagnostic imaging studies are necessary. Musculoskeletal symptoms can represent the major sign of the disease, leading to diagnostic difficulties. ${ }^{12}$ Musculoskeletal symptoms are common complaints, especially in the aging population. These similar symptoms also occur in up to $45 \%$ of patients with IE and are often misdiagnosed as rheumatic or degenerative joint disease. Arthralgia is the most common musculoskeletal complaint in patients with IE and involves shoulder most frequently, followed by knee, hip, wrist, ankle, and the joints of the hands and feet. Back pain is second in frequency, affecting almost a third of patients with IE. Usually, the patient presents with decreased range of motion and spinal tenderness. ${ }^{12}$ As regards the pathogenesis, some authors have suggested that back pain is due to microemboli of immune complexes with or without bacteria. ${ }^{15}$ The lumbar region is most commonly involved (60\%), and thoracic spine can also be affected (26\%). Neurologic deficits are usually not demonstrable in patients with back pain. ${ }^{16}$ Radiographic studies and bone scans confirm disease at the site, which may be caused by septic emboli. Common to all patients with IE, fever and heart murmur can be detected on physical examination and Gram-positive cocci can be isolated from blood cultures.

Naturally, the first choice must be the antibiotictherapy, both for IE and spondylodiscitis treatment. Cardiac valve replacement surgery should be performed if the course of fever and inflammatory syndrome is unfavorable after appropriate antibiotic treatment.

\section{CONCLUSION}

We conclude that, in all patients with spondylodiscitis, infective endocarditis should be ruled out, particularly in patients with a history of heart valve disease, since spondylodiscitis may be the presenting sign of an infective endocarditis. Echocardiography and blood cultures should be routinely performed in these patients. Cardiac valve replacement surgery should be performed if the course of fever and inflammatory syndrome is unfavorable after appropriate antibiotic treatment. We present a unique case of concomitant native valve endocarditis and spondylodiscitis caused by $S$. vestibularis. As the taxonomy of the viridans group becomes better defined, it is likely that novel disease associations will be made with the newly recognized species.

\section{REFERENCES}

1. Whiley RA, Hardie JM. Steptococcus vestibularis sp. nov. from the Human Oral Cavity. Int J Syst Bacteriol 1988; 38:335-9.

2. Cunliffe NA, Jacob A J. Streptococcus vestibulasis bacteraemia. J Infect 1997; 34;85-91.

3. Delorme C, Poyart C, Ehrlich SD, Renault P. Extent of horizontal gene transfer in evolution of streptococci of the salivarius group. J Bacteriol 2007; 189:1330-41.

4. Tunkel AR, Sepkowitz KA. Infections caused by viridans streptococci in patients with neutropenia. Clin Infect Dis 2002; 34:1524-9.

5. Wisplinghoff, Reinert RR, Cornely O, Seifert H. Molecular relationships and antimicrobial susceptibilities of viridans group streptococci isolated from blood of neutropenic cancer patients. Journal of Clinical Microbiology 1999; 37:1876-80.

6. Doyuk E, Ormerod OJ, Bowler IC. Native valve endocarditis due to Streptococcus vestibularis and Streptococcus oralis. J Infect 2002; 45:39-41.

7. Partridge SM. Prosthetic valve endocarditis due to Streptococcus vestibularis. J Infect 2000; 41:284-5.

8. Cone LA, Hirschberg J, Lopez C et al. Infective endocarditis associated with spondylodiscitis and frequent secondary epidural abscess. Surg Neurol 2008; 69:121-5.

9. Cahill DW, Love LC, Rechtine GR. Pyogenic osteomyelitis of the spine in the elderly. J Neurosurg 1991; 74:878.

10. Weber M, Gubler J, Fahrer H et al. Spondylodiscitis caused by viridans streptococci: three cases and a review of the literature Clin Rheumatol 1999; 18:417-21.

11. Beighton D, Hardie JM, Whiley RA. A scheme for the identification of viridans streptococci. J Med Microbiol 1991; 35:36772.

12. Le Moal G, Roblot F, Paccalin M et al.(2002) Clinical and laboratory characteristics of infective endocarditis when associated with spondylodiscitis. Eur J Clin Microbiol Infect Dis 2002; 21:671-5.

13. Ninet J, Gayet JL, Etienne J et al. Bacterial endocarditis presenting as acute vertebral osteomyelitis: 14 cases. Eur Heart J 1984; 5(Suppl):C101-5.

14. Thomas P, Allal J, Bontoux D et al. Rheumatological manifestations of infective endocarditis. Ann Rheum Dis 1984; 43:71620.

15. Morelli S, Carmenini E, Caporossi AP, Aguglia G. Spondylodiscitis and Infective endocarditis. Spine 2001; 26:499-500.

16. Hunder GG. When musculoskeletal symptoms point to endocarditis. J Musculosket Med 1992; 11:33-40. 